\title{
Spacetime and Reality: Facing the Ultimate Judge
}

\author{
Vesselin Petkov \\ Institute for Foundational Studies Hermann Minkowski \\ Montreal, Quebec, Canada \\ http://minkowskiinstitute.org/ \\ vpetkov@spacetimesociety.org
}

\begin{abstract}
Over a hundred years ago in his paper Space and Time Hermann Minkowski demonstrated the profound meaning of the relativity postulate - the experimental fact that physical phenomena are the same in all inertial reference frames implies that the Universe is an absolute four-dimensional world in which all moments of time have equal existence due to their belonging to the fourth (time) dimension. Since then there has been no consensus on the reality of this absolute world, which we now call Minkowski spacetime or simply spacetime. I will argue that facing the ultimate judge - the experimental evidence - allows us to settle this issue once and for all.
\end{abstract}

The views of space and time which I want to present to you arose from the domain of experimental physics, and therein lies their strength.

H. Minkowski [1]

\section{Introduction}

Over a century after the publication of Minkowski's paper Raum und Zeit in 1909 [2] the issue of the reality ${ }^{1}$ of spacetime (or the absolute world as Minkowski called it) - whether spacetime is just a mathematical four-dimensional space or it represents a real four-dimensional world - is still not resolved. What I think is even worse is that what seems to be the most shared view among both physicists and philosophers appears to be taking for granted the existence of some kind of objective becoming (which denies the reality of all events of spacetime) and thus denying the reality of the four-dimensional world, envisioned by Minkowski when he proposed the unification of space and time into an inseparable entity.

Explicitly or implicitly many physicists and philosophers regard spacetime as nothing more than a mathematical space which does not represent a real four-dimensional world. This view was openly defended by N. David Mermin in a recent article What's bad about this habit in the May 2009 issue of Physics Today where he argued that

\footnotetext{
${ }^{1}$ By "reality of spacetime" I mean, following Minkowski, a real four-dimensional world (not substantivalism).
} 
"It is a bad habit of physicists to take their most successful abstractions to be real properties of our world" [3]. He gave the issue of the reality of spacetime as an example - "spacetime is an abstract four-dimensional mathematical continuum" [3] - and pointed out that it is "a bad habit to reify the spacetime continuum" [3]. Although he did not make it entirely explicit, it is sufficiently clear from his article that part of his criticism was against substantivalism - he wrote: "I would urge you to consider that this continuum is nothing more than an extremely effective way to represent relations between distinct events". However, what is most relevant to the issue discussed here is his view that spacetime does not represent a real four-dimensional world: "The device of spacetime has been so powerful that we often reify that abstract bookkeeping structure, saying that we inhabit a world that is such a four- (or, for some of us, ten-) dimensional continuum" [3].

A hundred years after the publication of Minkowski's paper on spacetime it is difficult to explain the continued existence of views so clearly stating that spacetime does not have an ontological counterpart. By regarding spacetime as an abstract mathematical construction such views effectively ignore the revolutionary contribution of Minkowski to the advancement of our views of space and time. And indeed, if spacetime were merely a mathematical space with no counterpart in the world, we would call the unification of space and time Poincaré spacetime, not Minkowski spacetime, because it was Poincaré who first noticed in his paper Sur la dynamique de l'électron (before July 23, 1905 when he submitted the paper) that the Lorentz transformations had a geometric interpretation as rotations in what he seemed to have regarded as an abstract four-dimensional space [4, 168]. Poincaré appeared to have seen nothing revolutionary in the idea of a mathematical four-dimensional space since he believed that our physical theories are only convenient descriptions of the world and therefore it is really a matter of convenience and our choice which theory we would use. Here is Poincaré's own explanation [5]:

It quite seems, indeed, that it would be possible to translate our physics into the language of geometry of four dimensions. Attempting such a translation would be giving oneself a great deal of trouble for little profit, and I will content myself with mentioning Hertz's mechanics, in which something of the kind may be seen. Yet, it seems that the translation would always be less simple than the text, and that it would never lose the appearance of a translation, for the language of three dimensions seems the best suited to the description of our world, even though that description may be made, in case of necessity, in another idiom.

That Minkowski did not regard the absolute world implied by the relativity principle, as he argued, as an abstract four-dimensional continuum is seen even from the following general argument. Had he believed, like Poincaré, that uniting space and time into a four-dimensional space was only a convenient mathematical abstraction, he would not have written a paper whose title and content were devoted to something the main idea of which had already been published by Poincaré two years (and written more than three years) before Minkowski's talk on space and time given on September 
21, 1908, and would not have begun his paper with the now famous introduction, which unequivocally announced the revolution in our views on space and time: "From now onwards space by itself and time by itself will recede completely to become mere shadows and only a type of union of the two will still stand independently on its own" $[1]$.

However, the most convincing argument that Minkowski regarded the absolute four-dimensional world as real was provided by himself - he stressed that the strength of the new views of space and time he proposed comes from experimental physics [1]. So a century ago Minkowski was the first human (and please note - a mathematician) who faced the ultimate judge - the experimental evidence - on the issue of the ontological status of space and time. He did that by discussing one of the most important pieces of experimental evidence in the twentieth century - the negative result of the "famous interference experiment of Michelson" [1] that was carried out to detect the motion of the Earth with respect to the aether. Minkowski reviewed how Lorentz tried to explain it through a hypothesis that the arm of the interferometer used contracts in the direction of the Earth's motion due the contraction of its constituents (Lorentz used electrons to demonstrate the contraction hypothesis). Here is exactly how Minkowski realized that the experimental evidence forced upon us the new concept of space and time [1]:

Lorentz called $t^{\prime}$, which is a combination of $x$ and $t$, local time of the uniformly moving electron, and associated a physical construction with this concept for a better understanding of the contraction hypothesis. However, it is to the credit of A. Einstein who first realized clearly that the time of one of the electrons is as good as that of the other, i.e. that $t$ and $t^{\prime}$ should be treated equally. With this, time was deposed from its status as a concept unambiguously determined by the phenomena. The concept of space was shaken neither by Einstein nor by Lorentz ...

It was Minkowski who first made that attack on the concept of space when he realized that the postulated by Einstein equal reality of the time $t$ of an object believed to be at rest with respect to the aether and the time $t^{\prime}$ of an object in uniform motion was the profound physical meaning of the experimental impossibility to detect the motion of the Earth in the aether. Minkowski pondered over the implications of the fact that objects in relative motion have different (equally real) times and arrive at the inescapable conclusion - if there exist more than one time, there should exist more than one space as well. Minkowski explained [1] that in the case of two inertial reference frames in relative motion along their $x$-axes

one can call $t^{\prime}$ time, but then must necessarily, in connection with this, define space by the manifold of three parameters $x^{\prime}, y, z$ in which the laws of physics would then have exactly the same expressions by means of $x^{\prime}, y, z, t^{\prime}$ as by means of $x, y, z, t$. Hereafter we would then have in the world no more the space, but an infinite number of spaces analogously as there is an infinite number of planes in three-dimensional space. Three-dimensional geometry becomes a chapter in four-dimensional physics. You see why I 
said at the beginning that space and time will recede completely to become mere shadows and only a world in itself will exist.

Minkowski first realized the important hidden message in the experimental fact that physical phenomena are the same in all inertial reference frames (which Einstein merely stated in the relativity postulate without explaining it) - the experimental fact implies that the Universe is an absolute four-dimensional world in which space and time are inseparably amalgamated; only in such a world one can talk about many spaces and many times. And indeed, physical phenomena are the same in all inertial reference frames because every inertial observer describes the phenomena in his reference frame (i.e. in his own space and time) in which he is at rest. For example, the Earth is at rest with respect to its space and therefore all experiments confirm this state of rest. Minkowski noted that "I think the word relativity postulate used for the requirement of invariance under the group $G_{c}$ is very feeble. Since the meaning of the postulate is that through the phenomena only the four-dimensional world in space and time is given, but the projection in space and in time can still be made with a certain freedom, I want to give this affirmation rather the name the postulate of the absolute world" [1].

Since Minkowski arrived at the new view of space and time - that we live in an absolute four-dimensional world - when he faced the ultimate judge (a single experiment at that time), it is difficult to understand how what now appears to be a widespread view could still regard spacetime as nothing more than a mathematical continuum given the fact that we have at out disposal more experiments that confirmed the relativistic effects. A century after Minkowski's insight I think it will be fair to take our turn and also face the ultimate judge on the reality of spacetime. We owe this to Minkowski.

The main purpose of this paper is to demonstrate that the theory of relativity would be impossible if the world were three-dimensional. Sections 2 shows how relativity of simultaneity is possible only in a four-dimensional world. Section 3 revisits Minkowski's explanation of length contraction and discusses a more visualized version of his explanation through a thought experiment involving a meter stick, which demonstrates that no length contraction would be possible if the meter stick existed as a three-dimensional body.

\section{Relativity of Simultaneity}

Although no specially designed experiments have been carried out to test relativity of simultaneity, this major consequence of special relativity can be regarded as an experimental fact for two reasons:

- The experimental fact captured in the relativity postulate - physical phenomena are the same in all inertial reference frames - implies, as Minkowski demonstrated, that physical objects in relative motion have their own spaces and times, which can be explained if it is assumed that what exists is a four-dimensional world. But as a space constitutes a class of simultaneous events, it follows that 
relativity of simultaneity is a consequence of the existence of many spaces and therefore ultimately a consequence of the experimental fact of the invariance of physical phenomena, which is reflected in the relativity postulate.

- As we will see in Sections 3 length contraction, which have been experimentally confirmed, is a specific manifestation of relativity of simultaneity.

In order to understand fully why, unlike Poincaré, Minkowski appeared to have realized that special relativity would be impossible in a three-dimensional world, is to ask explicitly the questions that Minkowski appeared to have implicitly considered "What is the world?" and "How does the new concept of space and time affect our view of what exists?"

Concerning the first question, it seems we do not have much choice - most of those who regard spacetime as nothing more than a mathematical device appear (explicitly or implicitly) to hold the presentist view according to which the Universe is a single three-dimensional world defined as everything that exists simultaneously at the constantly changing moment 'now'. Minkowski might have started with this view too. Then, the answer to the second question follows naturally - the fact that objects in relative motion have different spaces (i.e. different classes of simultaneous events) implies that they have different three-dimensional worlds. But this is only possible if these worlds are different three-dimensional "cross sections" of an absolute four-dimensional world; it is impossible to have many spaces and times and relativity of simultaneity in a three-dimensional world. Indeed, if the world were three-dimensional (i.e., if spacetime were not representing a real four-dimensional world and were just an abstract mathematical space), there would exist one space and one absolute class of simultaneous events (since a three-dimensional space constitutes a single class of absolutely simultaneous events) in contradiction with relativity.

It should be stressed that the above argument is irrefutable only if the existence of physical objects and the world itself is regarded as absolute - only then the relativistic fact that observers in relative motion have different spaces and therefore different classes of simultaneous events implies an absolute four-dimensional world. But if existence is relativized, it appears to follow that relativity is possible in a three dimensional world - every observer would acknowledge the existence of only his own space (and three-dimensional world) and would deny the existence of the spaces (and the three-dimensional worlds) of the other observers in relative motion. So it appears that relativity of simultaneity (and therefore length contraction as well) can be explained either (i) in terms of absolute existence (in this case relativity of simultaneity implies a four-dimensional world - Minkowski's explanation) or (ii) in terms of relative existence (in this case the three-dimensionality of the world would be preserved what exists for each of the inertial observers in relative motion would be his relativized three-dimensional world).

It might be tempting to take the relativization of existence seriously since it preserves the pre-relativistic (presentist) view that what exists is a three-dimensional world; moreover, originally Einstein formulated special relativity in a three-dimensional language. But a careful analysis shows that that option contradicts the experiments 
that confirmed the twin paradox [6, Chap. 5]. In fact, it can be immediately shown that the idea of relativization of existence is not a serious alternative to the deep intuition that the very essence of existence makes it impossible to regard it as relative. If we assume that relativization of existence is the correct interpretation of relativity of simultaneity (which means that for every observer only his three-dimensional world would exist), we arrive at total nonsense when we ask what exists for an observer in general relativity.

Let us consider a single inertial observer and assume that what exists relative to the observer is his three-dimensional world, i.e. his present. In flat spacetime an inertial observer is represented by a straight worldtube and the presents corresponding to different moments of the proper time of the observer are parallel to one another and do not intersect. In curved spacetime, however, the worldtube of any observer is curved, which means that the presents at different moments of the observer's proper time intersect one another. As a result, some events which were past at a given moment would be future at a later moment. This nonsensical conclusion follows from the assumption that reality for an observer in general relativity is a three-dimensional world. Therefore considering even a single observer in general relativity rules out presentism and also its relativized version since the view that existence should be relativized regards the world of an observer as three-dimensional ${ }^{2}$.

After ruling out relativization of existence, the only way to interpret relativity of simultaneity (i.e. the fact that observers in relative motion have different spaces and times) is Minkowski's interpretation - that the classes of simultaneous events (i.e. the spaces) of observers in relative motion are "cross sections" of an absolute fourdimensional world. Let me stress that there is no alternative to this interpretation - if one assumed that spacetime were a mathematical device and that the Universe were a three-dimensional world, there would exist one (i.e. absolute) space and therefore one (i.e. absolute) class of simultaneous events in contradiction with the experimental evidence that physical laws are the same in all inertial reference frames.

In the sixties [7] and [8] pointed out that relativity of simultaneity does imply a four-dimensional world. In fact, their relativity of simultaneity argument, as we have seen, follows from the more general analysis (the existence of many spaces) that led Minkowski to the idea of spacetime. Like Minkowski's analysis, their argument has not been fully appreciated. Many physicists and philosophers have been refusing to accept the reality of the four-dimensional world of relativity, but have not explained how relativity of simultaneity would be possible if the world were three-dimensional. An example is Stein's criticism of the Rietdijk-Putnam argument [9], [10]. He correctly pointed out that one could not talk about distant present events in relativity but seemed to believe that he refuted the Rietdijk-Putnam argument (which, in essence, is Minkowski's argument) and some philosophers agreed with him. What he certainly refuted, however, is the presentist view according to which what exists is a single class of distant present events. Unfortunately, Stein criticized Rietdijk and Putnam for

\footnotetext{
${ }^{2}$ This argument can be also explained in the case of accelerated observers in special relativity [6, 150-152] since an accelerated observer is represented by a curved worldtube in flat spacetime and therefore the presents corresponding to different events of the observer's worldtube intersect.
} 
arguing that relativity of simultaneity implies a four-dimensional world, but explained neither how relativity of simultaneity would be possible if the world were not fourdimensional nor what the dimensionality of the world according to relativity would be.

So, when one explicitly asks the two questions above - what our view of the world is and how that view is affected by relativity - it follows that relativity of simultaneity is a manifestation of the four-dimensionality of the world (i.e. of the reality of spacetime) as Minkowski pointed out. Due the equal existence of all events of spacetime, observers in relative motion can regard different "cross sections" of it as their classes of simultaneous events (i.e. as their spaces). If there existed just one class of privileged (due to their existence) events all observers in relative motion would share that class and no relativity of simultaneity would be possible. When this is taken into account it becomes evident that the lack of an objectively privileged class of simultaneous events implies not only relativity of simultaneity, but also conventionality of simultaneity. This should be specifically emphasized since, I think, any claim that simultaneity is relative but not conventional would amount to a contradiction in terms - there exists no objectively privileged class of simultaneous events (due to relativity of simultaneity), but there exists an objectively privileged class of simultaneous events (due to the non-conventionality of simultaneity).

The relativity of simultaneity argument can be also used to rule out a theory proposed by Broad in 1923, which "accepts the reality of the present and the past, but holds that the future is simply nothing at all" [11]. Broad's growing (or evolving) block universe model of the world has been recently revived by several physicists [12], [13], [14] as what appears to be the last remaining alternative to the Minkowski absolute four-dimensional world. The recent versions of the growing block universe claim (excluding [13]) that they do not allow any form of a preferred structure. But if it is explicitly assumed that the existence of physical bodies is absolute, this claim cannot be supported. The hypersurface on the edge of the growing universe, on which the birthing (or coming into being) of events happens, constitutes an objectively privileged class of events (due to the absoluteness of existence), which contradicts relativity. Also, the growing block universe model leads to the same nonsense when used to explain what exists for an accelerated observer in flat spacetime or for an observer in curved spacetime.

\section{Length Contraction}

Length contraction was experimentally tested, along with time dilation, by the muon experiment in the muon reference frame (see for instance [15]). With this in mind, let us now ask whether this relativistic effect would be possible if a body, subjected to length contraction, were what we perceive - a three-dimensional object.

The essence of Minkowski's explanation of the deep physical meaning of length contraction of two bodies is that it is a manifestation of the reality of the bodies' worldtubes (Minkowski called them strips). This can be best understood from Fig. 1 


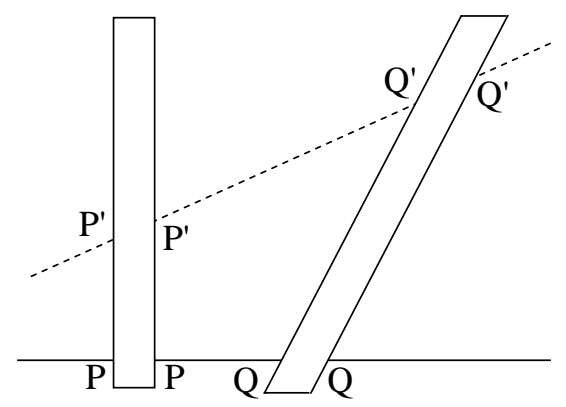

Figure 1: In Minkowski's paper Space and Time Fig. 1, part of which is reproduced here, represents two bodies or Lorentzian electrons by their worldtubes or as Minkowski called them (world) strips.

of his paper (the right-hand part of which is reproduced in Fig. 1 here) - length contraction would be impossible if the worldtubes of the two bodies, represented by the vertical and the inclined strips in Fig. 1, did not exist and were nothing more than "abstract geometric constructions" [3]. To see this even more clearly consider only the body represented by the vertical worldtube. The three-dimensional cross-section $P P$, resulting from the intersection of the body's worldtube and the space of an observer at rest with respect to the body, is the body's proper length. The three-dimensional cross-section $P^{\prime} P^{\prime}$, resulting from the intersection of the body's worldtube and the space of an observer moving with respect to the body, is the relativistically contracted length of the body measured by that observer. Minkowski stressed that "this is the meaning of the Lorentzian hypothesis of the contraction of electrons in motion" [1] and "that the Lorentzian hypothesis is completely equivalent to the new concept of space and time, which makes it much easier to understand" [1]. The worldtube of the body must be real in order that length contraction be possible because, while measuring the same body, the two observers in relative motion measure two three-dimensional bodies represented by the "cross-sections" $P P$ and $P^{\prime} P^{\prime}$ in Fig. 1.

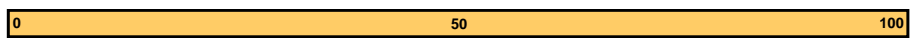

Figure 2: An ordinary meter stick.

This is not so surprising when one takes into account relativity of simultaneity and the fact that a spatially extended three-dimensional object is defined in terms of simultaneity - all parts of a body taken simultaneously at a given moment (so length contraction is indeed a specific manifestation of relativity of simultaneity). If the worldtube of the body were an abstract geometric construction and what existed were a single three-dimensional body (a single class of simultaneous events) represented by the proper cross-section $P P$, both observers would measure the same three-dimensional body, i.e. the same class of simultaneous events, which means that simultaneity would be absolute.

That length contraction of a body would be impossible if the body existed as a three-dimensional object (not a worldtube) can be perhaps better demonstrated by 
the following thought experiment, which is a more visualized version of Minkowski explanation. An ordinary meter stick (Fig. 2) is at rest with respect to an observer $A$. What is shown in Fig. 2 is what we perceive and take for granted that it is what really exists. According to Minkowski, however, the meter stick exists equally at all moments of its history and what is ultimately real is the worldtube of the meter stick as shown in Fig. 3.

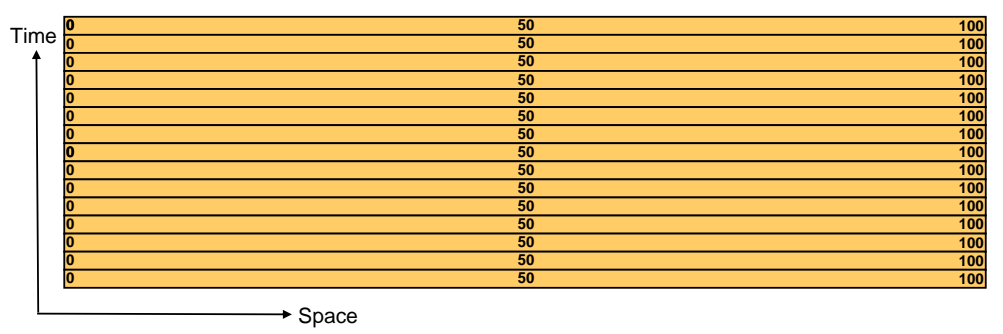

Figure 3: The worldtube of the meter stick.

Assume that another meter stick at rest in another observer's (observer B's) reference frame moves relative to the first one at a distance $1 \mathrm{~mm}$ above it. Let us assume that at the event $M$ the middle point of $B$ 's meter stick is instantaneously above the middle point of $A$ 's meter stick. Imagine also that lights are installed inside $A$ 's meter stick, which simultaneously change their color at every instant in $A$ 's frame. At the event of the meeting $M$ all lights are simultaneously red in $A$ 's frame. At all previous moments all lights were green. At all moments after the meeting all lights will be blue. When $A$ and $B$ meet at event $M$ this event is present for both of them. At that moment the present meter stick for $A$ is red (that is, all parts of $A$ 's meter stick, which exist simultaneously for $A$, are red). All moments before $M$ when all lights of the meter were green are past for $A$, whereas all moments when the meter stick will be blue are in $A$ 's future.

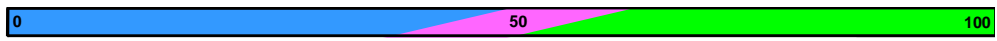

Figure 4: Relativistically contracted meter stick measured by observer $B$.

Imagine that, instead of lights, $B$ 's meter stick contains cameras at every point along its length. At the events of the meeting $M$ all cameras take snapshots of the parts of $A$ 's meter stick which the cameras face. All snapshots are taken simultaneously in $B$ 's reference frame.

Even without looking at the pictures taken by the cameras, it is clear that not all pictures will show a red part of $A$ 's meter stick, because what is simultaneous for $A$ is not simultaneous for $B$. When $B$ assembles the picture of $A$ 's meter stick from the pictures of all cameras it would show two things as depicted in Fig 4 - (i) $A$ 's meter stick photographed by $B$ is shorter, and (ii) only the middle part of the picture of $A$ 's meter stick is red; half is green and the other half is blue. So what is past (green), present (red), and future (blue) for $A$ exists simultaneously as present for $B$. But this is only possible if the meter stick is the worldtube as shown in Fig. 5. The instantaneous space of $B$ corresponding to the event $M$ intersects the worldtube of 
the meter stick at an angle and the resulting three-color "cross section" is what is measured by $B$ - a different three-dimensional meter stick, which is shorter ${ }^{3}$ than the meter stick measured by $A$.

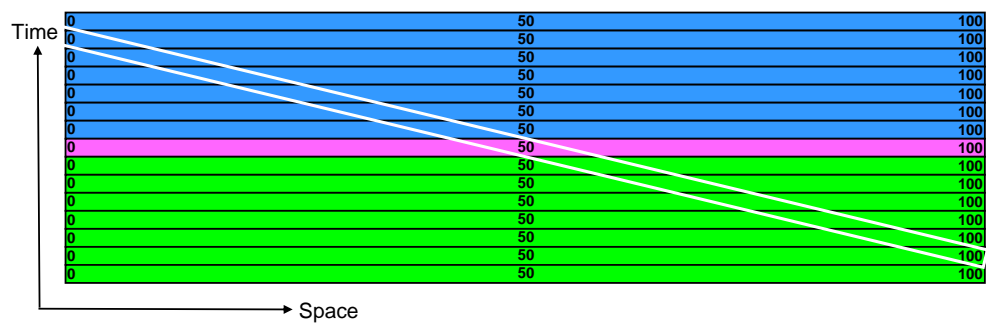

Figure 5: The worldtube of the meter stick with different colors.

It should be stressed as strongly as possible that no length contraction would be possible if the meter stick's worldtube did not exist as a four-dimensional object. If the meter stick were a three-dimensional object, both observers would measure the same three-dimensional meter stick (the same set of simultaneously existing parts of the meter stick), which would mean that the observers would share the same (absolute) class of simultaneous events in a clear contradiction with relativity [16].

As length contraction implies that physical objects are worldtubes it would provide further support to the arguments that relativity favours (i) perdurantism over endurantism [17], and (ii) the worm theory over the stage theory [18].

\section{Conclusion}

Minkowski arrived at the new concept of space and time by analyzing a single experiment - the impossibility to detect the motion of the Earth with respect to the aether. A century after him it is our turn to face the greater number of relativistic experiments which are at our disposal. Instead of leaving the resolution of the issue of the reality of spacetime to the next generations we can start working on its implications for science, philosophy, and our entire world view. Refusing to face the implications of Minkowski's view because of the huge challenges they pose, and trying to squeeze Nature into our pre-set and deceivingly comfortable views of the world should not be an option for anyone in the 21st century.

\section{References}

[1] H. Minkowski, "Space and Time." New translation in V. Petkov (ed.), H. Minkowski, Space and Time: Minkowski's Papers on Relativity, (Minkowski Institute Press, Montreal 2012).

\footnotetext{
${ }^{3}$ In Fig. 5 the inclined "cross section," which represents the different three-dimensional meter stick measured by $B$, appears longer, not shorter, because a fact in the pseudo-Euclidean geometry of spacetime is represented on the Euclidean surface of the paper.
} 
[2] H. Minkowski, "Raum und Zeit." Physikalische Zeitschrift 10 (1909) pp. 104-111; Jahresbericht der Deutschen Mathematiker-Vereinigung 18 (1909) pp.75-88.

[3] N.D. Mermin, "What's Bad About This Habit?" Physics Today 2009, p. 8.

[4] H. Poincaré, "Sur la dynamique de l'électron." Rendiconti del Circolo matematico Rendiconti del Circolo di Palermo 21 (1906) pp.129-176.

[5] H. Poincaré, Science and Method, In: The Value of Science: Essential Writings of Henri Poincaré (Modern Library, New York 2001), p. 438

[6] V. Petkov, Relativity and the Nature of Spacetime, 2nd ed. (Springer, Heidelberg 2009).

[7] C.W. Rietdijk, "A Rigorous Proof of Determinism Derived from the Special Theory of Relativity." Philosophy of Science 33 (1966) pp. 341-344.

[8] H. Putnam, "Time and Physical Geometry." Journal of Philosophy 64 (1967) pp. 240-247.

[9] H. Stein, "On Einstein-Minkowski Space-time." Journal of Philosophy 65 (1968) pp. 5-23.

[10] H. Stein, "On Relativity Theory and the Openness of the Future." Philosophy of Science 58 (1991) pp. 147-167.

[11] C.D. Broad, Scientific Thought (Littlefield, Adams and Co, New Jersey 1959).

[12] J. Christian, "Absolute Being versus Relative Becoming." In V. Petkov (ed.) Relativity and the Dimensionality of the World, (Springer, Heidelberg 2007) pp. 163-195. Arxiv:gr-qc/0610049.

[13] G.F.R. Ellis, "Physics in the Real Universe: Time and Space-Time." In V. Petkov (ed.) Relativity and the Dimensionality of the World, (Springer, Heidelberg 2007), pp. 49-79. Arxiv:gr-qc/0605049.

[14] R.D. Sorkin, "Relativity theory does not imply that the future already exists." In V. Petkov (ed.) Relativity and the Dimensionality of the World, (Springer, Heidelberg 2007), pp. 153-161. Arxiv:gr-qc/0703098.

[15] G.F.R. Ellis and R.M. Williams, Flat and Curved Space Times (Oxford University Press, Oxford 1988) p. 104.

[16] V. Petkov, "On the Reality of Minkowski Space." Foundations of Physics 37 (2007) pp. 1499-1502.

[17] S.D., Hales and T. Johnson, "Endurantism, Perdurantism, and Special Relativity." The Philosophical Quarterly 53 (2003) pp. 524-539.

[18] Y. Balashov, "On Stages, Worms, and Relativity." In C, Callender (ed.) Time, Reality, and Experience (Cambridge University Press, Cambridge 2002) pp. 223252 . 